\title{
Development of an efficient and resilient algorithm For lane feature extraction in image sensor-based lane de- tection
}

\author{
Yung-Hsiang Liu ${ }^{1}$, H. P. Hsu ${ }^{2}$, S. M. Yang ${ }^{3 *}$ \\ ${ }^{1,3}$ Department of Aeronautics and Astronautics Engineering, National Cheng Kung University, Cheng Kung, Taiwan \\ ${ }^{2}$ International Degree Program on Energy Engineering National Cheng Kung University, Cheng Kung, Taiwan
}

\section{Keywords \\ Lane detection \\ Inverse perspective mapping \\ Line segment detector \\ Random sample consensus}

Received: 13 February 2019

Accepted: 14 March 2019

Published: 20 April 2019

\begin{abstract}
Lane detection is key to advanced driver assistance systems to avoid traffic accidents caused by driver's negligence, thereby improving driver's safety. However, most lane detection algorithms are prone to error in challenging conditions when maneuvering in high curvature lanes, strong backlighting environment, low contrast night, and heavy rain condition, rendering unreliable and hazardous detection motion. This work proposes a lane detection algorithm by robust binary lane marking identification for lane feature extraction. The algorithm combines the median local threshold, line segment detector, and binary line segment filter to remove the noise generated when operating in the above challenging conditions. After correct lane feature extraction, Hough transformed and optimized random sample consensus parabola fitting are applied to detect lane markings. Experiment results show that the proposed algorithm outperforms the previous work in achieving correct detection rate at $95 \%$ in real-time.
\end{abstract}

(c) 2019 The Author(s). Published by TAF Publishing.

\section{INTRODUCTION}

Unintended lane departure due to driver's negligence has been known as the leading cause of increasing vehicle accidents. Advanced Driver Assistance Systems (ADAS) are considered pivotal to reduce accident and improve safety $[1,2]$. The reliability of system is dependent on the results of lane detection, where process time and robustness are key to the success of ADAS. Most lane detection results focus only on cost-effective image sensor (dash-cam) for sensors such as Light Detection and Ranging (LIDAR) and Radio Detection and Ranging (RADAR) are not available in publicTransport as yet. Although vision-based lane detection is widely adopted for future ADAS, it suffers from the noise generated by challenging conditions such as high curvature, strong backlighting, low contrast night, and heavy rain $[3,4]$. Therefore, the robustness of lane detection becomes an important issue. It determines whether an ADAS can be applied with strong confidence.
For vision-based algorithms in the last decade, [5] adopted the $[6,7]$ to extract lane features and the possible angle range of the lanes (according to the perspective of a fixed camera on the vehicle) to remove undesirable noise in the image. [8] proposed a geometric rule-based lane detection with two-stage feature to filter false lane features. Though the above algorithms are helpful to improving lane detection, the computational complexity is increased dramatically. In this regard, [9] introduced a symmetrical local threshold [10] based on the limitations of marking width to extract lane features, and a Bresenham line voting space is then presented to detect lanes at low-speed environments. [11] proposed a simplified algorithm to detect lanes in high curvature. [12] concluded that a lane detection algorithm should be robust to detect lane markings in all challenging conditions and to respond for dangerous situations immediately. This work proposes a robust algorithm based on

\footnotetext{
${ }^{*}$ Corresponding author: S. M. Yang

†email: smyang@mail.ncku.edu.t
} 
binary line segment filter for detecting lanes by using only a low-cost image sensor. The algorithm combines the median local threshold [13], the line segment detector [14], and the proposed binary line segment filter to remove image noises and to extract lane features. The lane marking is then model by Hough transform [15] and random sample consensus parabola fitting [16] to detect lanes with high efficiency for real-time applications.

\section{ALGORITHM ARCHITECTURE BY IMAGE SENSOR}

A typical image-based lane detection algorithm has three stages: image preprocessing, lane feature extraction, and lane model fitting as illustrated in Figure 1. Image preprocessing is to convert a frontal view of RGB image into a bird's eye view of the grayscale image by inverse perspective mapping, thereby reducing the sensor error. Lane feature extraction is to extract line segments as candidate line segments of lane marking. The binary line segment filter is developed in this work to reduce the noise in the candidate line segments. Lane model fitting is by the simplified Hough transform and by an optimized Random Sample Consensus (RANSAC) parabola fitting to accurately detect the lane markings in challenging conditions.

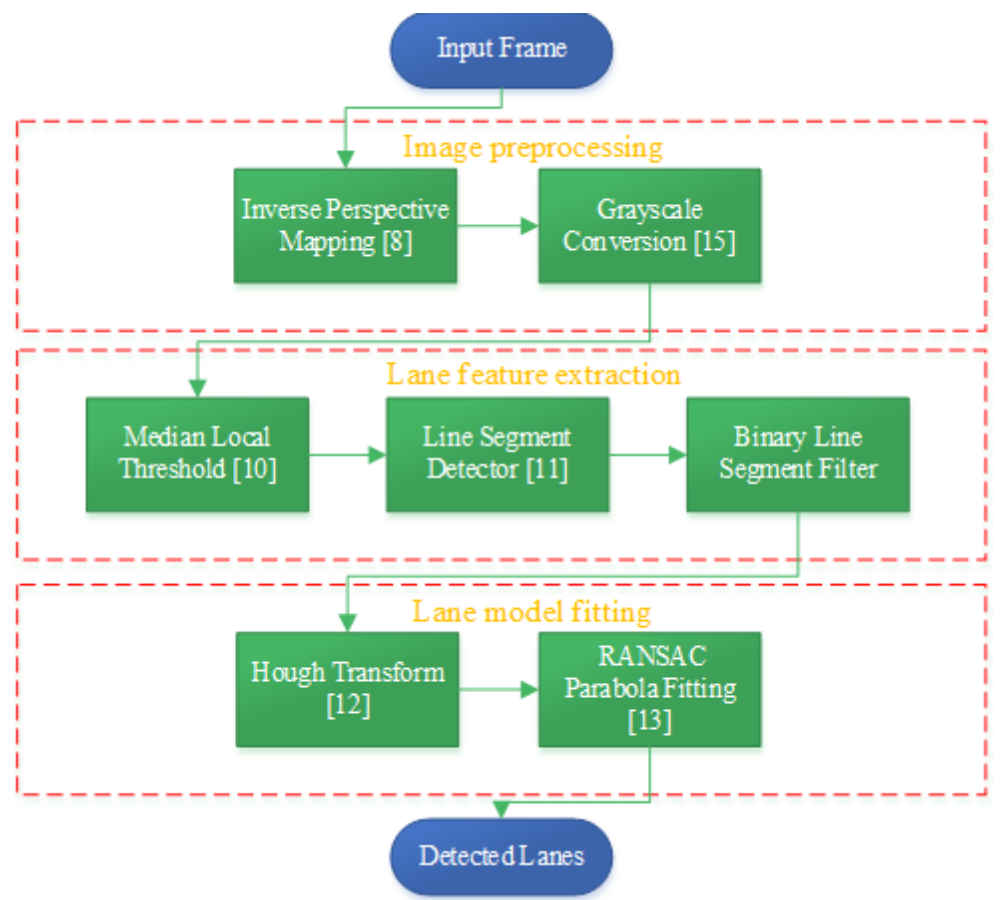

Fig. 1. The lane detection algorithm proposed in this work, where the image preprocessing is to reduce the sensor error, the lane feature extraction is to reduce noise, and the lane model fitting is to fit the lane markings in the image

\section{A. Image Preprocessing}

The input images to lane detection algorithm are collected from an image sensor (dash-cam) mounted on the car as illustrated in Figure 2 with the world coordinates $\left(X_{w}, Y_{w}, Z_{w}\right)$ and the camera coordinates $\left(X_{c}, Y_{c}, Z_{c}\right)$. To correct the image affected by perspective effect, it is transformed from a frontal-view image to a bird's eye view image. The lane markings in bird's eye view images becomes parallel and the widths are approximately equal. The conversion from an arbitrary point $P_{w}\left(X_{w}, Y_{w},-h\right)$ of the world coordinates to the corresponding point $P_{i}\left(u_{i}, v_{i}\right)$ of the image plane can be determined by coordinate transformation by the focal length $\left(f_{u}, f_{v}\right)$ and the camera optical center $\left(c_{u}, c_{v}\right)$ of the image sensor.

$$
u_{i}=\frac{c_{u}+f_{u}\left(X_{w} \cos \theta-Y_{w} \sin \theta\right)}{X_{w} \cos \alpha \sin \theta+Y_{w} \cos \alpha \cos \theta+h \sin \alpha}
$$

(2)

$v_{i}=\frac{c_{v}+f_{v}\left(-X_{w} \sin \alpha \sin \theta-Y_{w} \sin \alpha \cos \theta+h \cos \alpha\right)}{X_{w} \cos \alpha \sin \theta+Y_{w} \cos \alpha \cos \theta+h \sin \alpha}$

where $\alpha$ is the pitch angle and $\theta$ is the yaw angle. Figure 3 (a) and (b) illustrate an input image transformed to a bird's eye view image, which is then converted to a grayscale image [17]. 

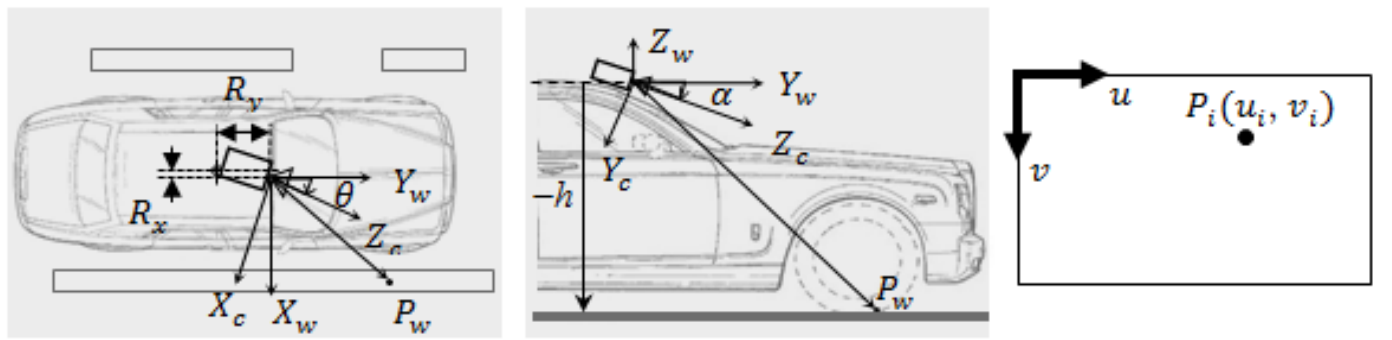

Fig. 2. (a) An image sensor (dash-cam) with the camera coordinates $\left(X_{c}, Y_{c}, Z_{c}\right)$ mounted on a vehicle at height $h$ above ground with pitch angle $\alpha$, yaw angle $\theta$, and the offset $\left(R_{x}, R_{y}\right)$ from the vehicle center in the world coordinates $\left(X_{w}, Y_{w}, Z_{w}\right)$, and (b) illustration of the image plane, where point $P_{i}$ is the projection from a point $P_{w}$ on the ground to the image plane.

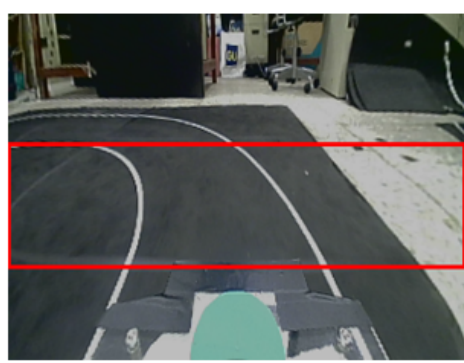

(a) Input image

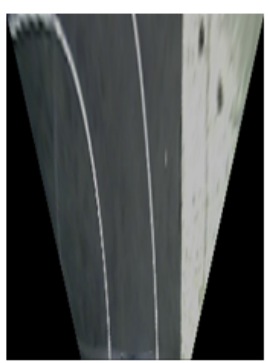

(b) Bird's eye view image

Fig. 3. (a) Application of the inverse perspective mapping to transform a region of interest (in box) to (b) the bird's eye view image

\section{B. Lane Feature Extraction by Binary Line Segment Fil- ter}

In poor condition of backlighting, night, or rain, the lane marking becomes blurred, so that extracting the lane feature is challenging. This work adopts the median local threshold [13] to extract the preliminary lane features. The median local threshold uses a 1D fast median filter [18] to conduct line-by-line filtering of the grayscale image. If the intensity of a point differs from the median by more than a given threshold, then the point is considered a lane feature.

$$
I_{m}\left(u_{i}, v_{i}\right)=\left\{\begin{array}{cc}
I\left(u_{i}, v_{i}\right), & \text { if } I\left(u_{i}, v_{i}\right)>\operatorname{median}\left(I\left(u_{i}, S_{m}\right)\right)+T_{g} \\
0, & \text { otherwise }
\end{array}\right.
$$

where $I_{m}$ is a grayscale image processed by a median local threshold, $S_{m}$ is the 1D median filter determined by the width of the lane marking, $T_{g}$ is the threshold given in the lane detection algorithm. Figure 4 (a) and (b) illustrate the grayscale image before and after the median local threshold, which significantly reduces unnecessary information (such as tarred road and curb), and the intensity and geometry of the lane markings are preserved in the lane features.

However, the processed grayscale image still has some false lane features geometrically similar to lane markings. These false lane features are mainly caused by the pedestrian crossing, stain, and vertical component from vehicles. A lane marking of any curvature can be approximated as a combination of line segments, so that the false lane features in the geometric structure of a lane marking can be eliminated by applying the line segment detector [14]. The detector starts by calculating the level-line angle of each pixel to form a level-line field in the image. The level-line field is then divided into the line support region of the same angle range as illustrated in Figure 5 (a).

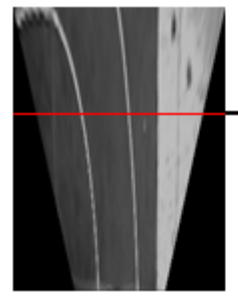

(a)

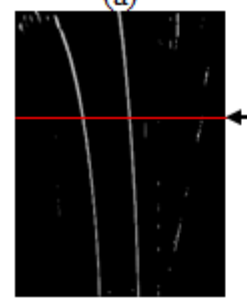

(d)

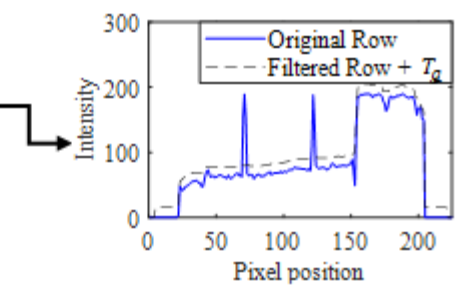

(b)
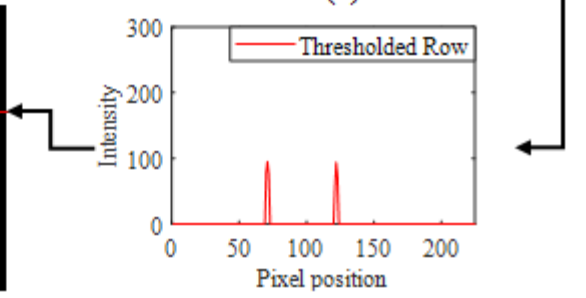

(c)
Fig. 4. (a) The grayscale image before the median local threshold processing, (b) the intensity distribution of a row (solid line) after the median local threshold processing, (c) the intensity distribution of a row (dotted line) retained by the threshold, and (d) the grayscale image after the median local threshold processing 
Each line support region is a candidate for generating a binary line segment. Through the above process, the grayscale image is converted to a binary image as shown in Figure 5 (b), where the noise of too short and/or incorrect tangent in a segment can be eliminated by the binary line segment filter based on geometric constraints,

$$
\text { Rule: } \tau_{i}>T_{1} \text { and } T_{L} \leq \vartheta_{i} \leq T_{U}
$$

where $\tau_{i}$ and $\vartheta_{i}$ are the length and angle of each line segment as shown in Figure 5 (c), respectively, $T_{1}$ is the fixed threshold determined by the length distribution histogram, and $T_{L}$ and $T_{U}$ are the adaptive threshold. The binary image before and after processing by the above rule are shown in Figure 6, where the noise line segments have been eliminated.

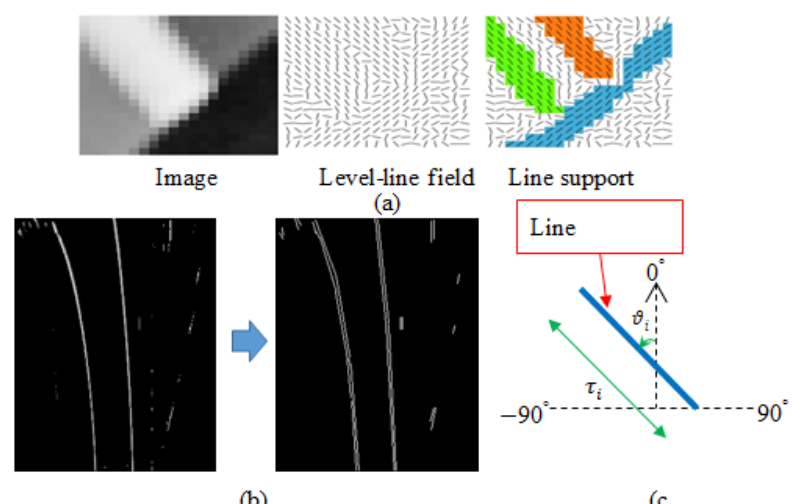

(b)

(c

Fig. 5. (a) The schematic diagram of generating a linesupport area by first calculating the level-line angle of each pixel in the image, and then generating a linesupport region at the adjacent pixel point as a line segment, (b) the binary image before and after the line segment detector processing (left and right), and (c) calculating the length and angle of the line segment, where $\tau_{i}$ and $\vartheta_{i}$ are the length and angle of the line segment (thick line), respectively.

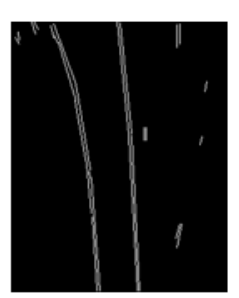

(a)

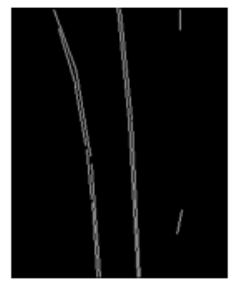

(b)

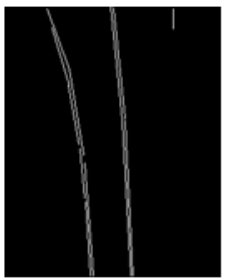

(c)
Fig. 6. (a) The binary image before the binary line segment filter processing, (b) the first rule based on the length of line segment is used to eliminate too short line segment, and (c) the second rule based on the angle of line segment is used to eliminate line segment with abnormal slope in the binary image

\section{Lane Model Fitting}

With the extracted lane feature, it is necessary to detect the position of the left and/or right lane marking for lane model fitting. This work adopts a simplified Hough transform with sliding window [15]. Due to the lane markings close to the vehicle are approximately vertical in the bird's eye view image, the simplified Hough transform calculates the column intensity sum, where the local maximum is detected simultaneously to locate the horizontal positions $x_{L}$ and $x_{R}$ of the left/right lane markings and the lane width $\mathrm{w}$ as shown Figure 7 (a). The horizontal position of the lane marking is originally located as the starting position of the center of the first window, and then the pixels mean position in the first window is calculated as the center position of the next window. In order to effectively fit the lane markings with minimal computation load, a quadratic parabola model with optimized RANSAC [16] is applied to fit the lane marking. The pixels in the previous sliding window are segmented into sub-sets based on the number of candidate points in the sliding window to be evenly distributed to each subset. A point from each subset is randomly selected to define the parabola geometry by least square. The iterative process is to calculate the score for each parabola fit and the highest score for the lane model fitting as shown in Figure 7 (c).

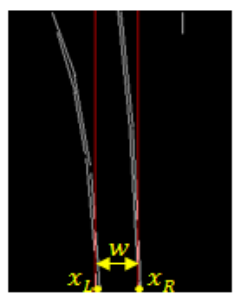

(a)

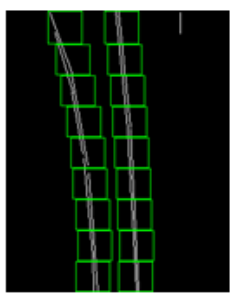

(b)

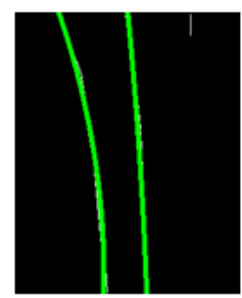

(c)
Fig. 7. (a) The column intensity sum of the binary image with peaks at the left and right lane markings $x_{L}$ and $x_{R}$ in the horizontal direction, where the distance between the above two positions are the lane width $w$, (b) the simplified Hough transform by locating the position of the left and right lane markings to build a series of fixed-size sliding windows, and (c) the optimized RANSAC parabola fitting to fit the lane markings

\section{EXPERIMENTAL VERIFICATION}

In the experiment, the robustness of the algorithm with only an image sensor is verified by 297 testing images in challenging conditions. An image sensor (dash-cam) with $140^{\circ}$ Field of View (FOV) and 30 Frames Per Second (FPS) is mounted on the car at height $h=21.3 \mathrm{~cm}$ above the ground plane; yaw angle $\theta=0^{\circ}$, pitch angle $\alpha=20^{\circ}$, and offset $\left(R_{x}, R_{y}\right)=(0,12.5)$ from the vehicle as shown in Figure 2 . In order to ease the computational complexity of the algo- 
rithm, this work sets the image range containing the mostly road information to the Region of Interest (ROI) of width 319 pixels and height 85 pixels with the position of the top left corner $(0,12.5)$ in the input image $(320 \times 240$ pixels $)$ as shown in Figure 3 (a). By applying the Inverse Perspective Mapping (IPM), the input image is transformed to the bird's eye view image $(225 \times 300$ pixels $)$, so that the lane markings becomes parallel as shown in Figure 3 (b). The bird's eye view image is then converted to grayscale image for improving detection upon facing various lane markings (such as discontinuous, white, and yellow lane markings) as shown in Figure 4 (a). The intensity histogram is calculated from the grayscale image with the dotted line marking the threshold determined by the median local threshold as shown in Figure 4 (b). The grayscale image after the median local threshold processing is converted to the binary image by the line segment detector, which can extract all line segments to highlight the geometric structure of the lane markings for later analysis as shown in Figure 5 (b).

The proposed binary line segment filter based on geometric rule is then used to eliminate noise line segment in the binary image. According to the length distribution histogram of the line segment, the length of noise line segments are mostly less than 17 in all testing images as shown in Figure 8.

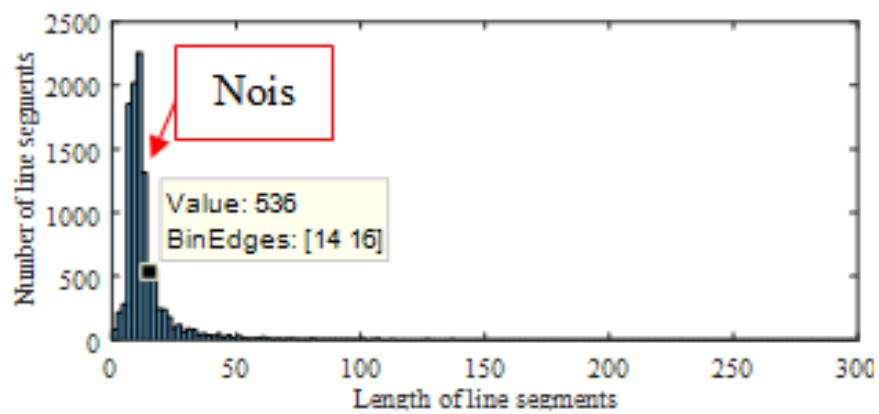

(a)
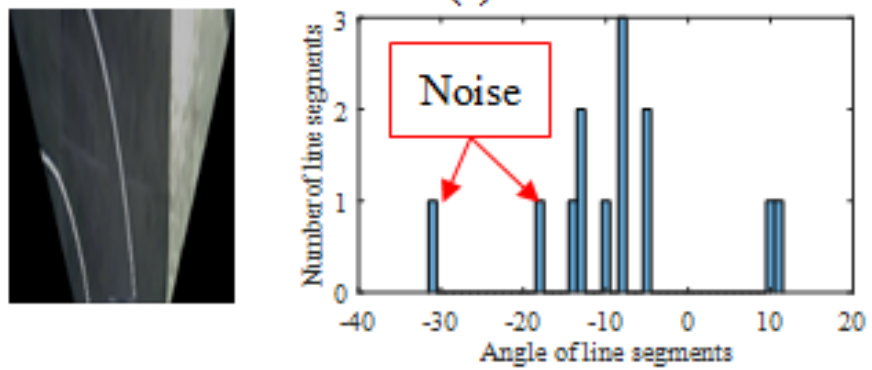

(b) Left curved lane
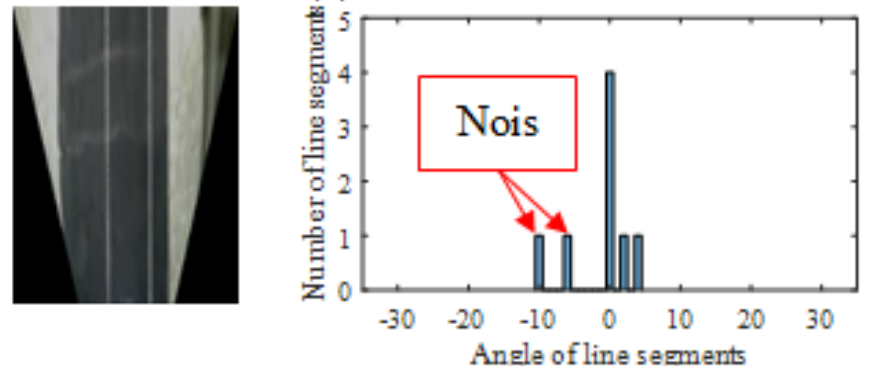

\section{(c) Straight}

Fig. 8. The histogram analysis to determine the threshold of the rule of the binary line segment filter, (a) the histogram is the length distribution of the line segments produced by the images collected in the experiment, where length of the noise line segment is mostly less than 17 , (b) the image of left curved lane with the histogram shows the angular distribution of the line segment in-35 $0^{\circ}$, and (c) the image of straight lane with the histogram of the angle distribution of the line segment in $-5 \sim 5^{\circ}$. 
The binary image before and after processing by the above geometric relationship are shown in Figure 6 (a) and (b), where the noise line segments of too short can be eliminated. According to the angular distribution generated by the lane marking, each line segment is assigned to the specified angle region according to the calculated angle, and the calculated length is accumulated as a score. The line segment of lane marking in different road scenes and their corresponding angular distribution histograms are indicated in Figure 8 (b) and (c). Through the histogram, the binary image before and after processing by the above geometric relationship are shown in Figure 6 (b) and (c), where the noise line segments with abnormal slope can be eliminated. The column intensity sum of the binary image can be calculated to locate the horizontal position $x_{L}$ and $x_{R}$ of the left and right lane markings and the lane width " $w$ " as shown in Figure 7 (a). A series of fixed size sliding windows are then built on the bottom of each lane marking as shown in Figure 7 (b) and (c) for minimal computation load on the on-board computer.
Experimental results of lane feature extraction before and after using binary line segment filter validate that the lane detection algorithm can effectively reduce the number of false lane features. In addition, Table 1 shows the performance between the lane detection algorithm with and without binary line segment filter to verify the effectiveness of the algorithm. Among them, the correct rate of the proposed algorithm without binary line segment filter in the above four conditions is $97 \%, 89 \%, 92 \%$, and $78 \%$, while the false rate is $3 \%, 11 \%, 8 \%$, and $10 \%$, respectively. After using the binary line segment filter, the correct rate of the proposed algorithm with binary line segment filter in the above four conditions is $99 \%, 93 \%, 92 \%$, and $95 \%$, respectively. The correct rate by using the binary line segment filter is significantly improved, especially in heavy rain. Moreover, this paper also performs the same test on the algorithm proposed by [11], where the results show that the correct rate is easily affected by the noise to the lane marking in challenging conditions, especially the strong backlighting because they only adopted the intensity information of the lane marking for detecting lanes.

TABLE 1

RESULTS OF LANE DETECTION ALGORITHM OF THIS WORK WITH AND WITHOUT BINARY LINE SEGMENT FILTER (BLSF) [?]

\begin{tabular}{llll}
\hline \hline Challenging Conditions & \multicolumn{3}{l}{ Correct rate (\%) } \\
\cline { 2 - 5 } & {$[11]$} & This Work without BLSF & This Work with BLSF \\
\hline High curvature & 19 & 97 & 99 \\
Strong backlighting & 3 & 89 & 93 \\
Low contrast night & 36 & 92 & 92 \\
Heavy rain & 17 & 78 & 95 \\
\hline \hline
\end{tabular}

For a series of 297 captured images of a typical maneuver in challenging conditions, the average computation time of lane detection by the on-board computer with CPU (ARM, 2 $\mathrm{GHz}$ ) is about $32.7 \mathrm{~ms}$. Through the above results of verification, the proposed lane detection algorithm can be proved to meet the robustness and immediacy of the ADAS requirement. This algorithm is critical to the development of autonomous delivery systems.

\section{SUMMARY AND CONCLUSION}

In order to evaluate the performance of the lane detection algorithm, this work adopts the evaluation index [19] to calculate the correct rate of the lane detection algorithm. The two guidelines: (1) when at least more than half lane marking estimates are within the lane marking width of the ground-truth and, (2) when no lane marking is existent, yet one is detected as incorrect detection. To validate the robustness of the lane detection algorithm, this work tests the images of four challenging conditions: high curvature, strong backlighting, low contrast night, and heavy rain. Table 1 shows that the average correct rate of the proposed lane detection algorithm in the above four conditions is $95 \%$. Compared with the lane detection of previous work [11], the results also show that the proposed lane detection is effective and resilient as in Table 1 . The lane detection proposed in this work can better detect the lane marking in challenging conditions, because the algorithm has fully utilized the intensity and geometric property of the lane markings to develop the novel feature extractor for detecting lanes. The conclusions are:

(1) This work proposes a robust lane detection algorithm based on median local threshold, line segment detector, and the binary line segment filter. The median local threshold and the line segment detector are combined to obtain all line segments in the image for a preliminary lane feature extraction. Then, the binary line segment filter based on ge- 
ometric rule is to eliminate noise line segments. The lane feature extraction is tested to show that the proposed binary line segment filter can effectively reduce noise in the image to improve the correct rate of detection.

(2) With effective lane feature extraction, a simplified Hough transform with sliding window and an optimized RANSAC parabola fitting are employed to fit the lane markings in challenging conditions. The lane detection algorithm is tested by road images collected from experiment, including high curvature, strong backlighting, low contrast night, and heavy rain. The test results indicate that the average correct rate of the proposed lane detection algorithm is $95 \%$, and the average computation time per image is about
$32.7 \mathrm{~ms}$. These results prove that the proposed lane detection algorithm is capable of handling challenging conditions in real-time for ADAS and autonomous delivery systems.

(3) The proposed lane detection algorithm is effective, but the input image must contain at least one lane marker for detection in this work. For future development, this work can add the lane tracking to improve the efficiency of the lane detection. The lane tracking uses currently known lane marking position to predict at the next position, rendering the detection result is not susceptible to interference of road scenes without lane markings in a short time (such as crosswalk and worn road) and to reduce the computation time required for detecting the lane markings.

\section{REFERENCES}

[1] J. Son, H. Yoo, S. Kim, and K. Sohn, "Real-time illumination invariant lane detection for lane departure warning system," Expert Systems with Applications, vol. 42, no. 4, pp. 1816-1824, 2015. doi: https://doi.org/10.1016/j.eswa.2014.10.024

[2] F. Gongor, O. Tutsoy, and S. Colak, "Development and implementation of a sit-to-stand motion algorithm for humanoid robots," Journal of Advances in Technology and Engineering Research, vol. 3, no. 6, pp. 245-256, 2017. doi: https://doi. org/10.20474/jater-3.6.4

[3] Y. Xing, C. Lv, L. Chen, H. Wang, H. Wang, D. Cao, E. Velenis, and F.-Y. Wang, “Advances in vision-based lane detection: Algorithms, integration, assessment, and perspectives on ACP-based parallel vision," IEEE/CAA Journal of Automatica Sinica, vol. 5, no. 3, pp. 645-661, 2018. doi: https://doi.org/10.1109/jas.2018.7511063

[4] L. Jeeyoung, G. Habimana, H. Kyungbo, K. T. Jeong, and K. Sunkuk, "Panelizing algorithms for free-form concrete panels considering esthetic surfaces," International Journal of Technology and Engineering Studies, vol. 1, no. 3, pp. 81-86, 2015. doi: https://doi.org/10.20469/ijtes.40003-3

[5] D. C. Andrade, F. Bueno, F. R. Franco, R. A. Silva, J. H. Z. Neme, E. Margraf, W. T. Omoto, F. A. Farinelli, A. M. Tusset, S. Okida et al., "A novel strategy for road lane detection and tracking based on a vehicle's forward monocular camera," IEEE Transactions on Intelligent Transportation Systems, vol. 20, no. 4, pp. 1497-1507, 2018. doi: https://doi.org/10. 1109/tits.2018.2856361

[6] J. Canny, "A computational approach to edge detection," IEEE Transactions on Pattern Analysis and Machine Intelligence, no. 6, pp. 679-698, 1986. doi: https://doi.org/10.1109/tpami.1986.4767851

[7] A. H. Alhussain, "Using deterministic genetic algorithm to provide secured cryptographic pseudorandom number generators," International Journal of Technology and Engineering Studies, vol. 1, no. 4, pp. 107-116, 2015. doi: https: //doi.org/10.20469/ijtes.40001-4

[8] J. Niu, J. Lu, M. Xu, P. Lv, and X. Zhao, "Robust lane detection using two-stage feature extraction with curve fitting," Pattern Recognition, vol. 59, pp. 225-233, 2016. doi: https://doi.org/10.1016/j.patcog.2015.12.010

[9] Q. Li, J. Zhou, B. Li, Y. Guo, and J. Xiao, "Robust lane-detection method for low-speed environments," Sensors, vol. 18, no. 12, pp. 42-74, 2018. doi: https://doi.org/10.3390/s18124274

[10] U. Ozgunalp and N. Dahnoun, "Robust lane detection \& tracking based on novel feature extraction and lane categorization," in IEEE International Conference on Acoustics, Speech and Signal Processing (ICASSP), Florence, Italy, 2014.

[11] C. Kuo, Y. Lu, and S.-M. Yang, "'On the image sensor processing for lane detection and control in vehicle lane keeping systems," Sensors, vol. 19, no. 7, pp. 16-65, 2019. doi: https://doi.org/10.3390/s19071665

[12] S. P. Narote, P. N. Bhujbal, A. S. Narote, and D. M. Dhane, "A review of recent advances in lane detection and departure warning system," Pattern Recognition, vol. 73, pp. 216-234, 2018. doi: https://doi.org/10.1016/j.patcog.2017.08.014

[13] E. Pollard, D. Gruyer, J.-P. Tarel, S.-S. Ieng, and A. Cord, "Lane marking extraction with combination strategy and comparative evaluation on synthetic and camera images," in 14th International IEEE Conference on Intelligent Transportation Systems (ITSC), California, CA, 2011. 
[14] R. G. Von Gioi, J. Jakubowicz, J.-M. Morel, and G. Randall, "LSD: A fast line segment detector with a false detection control," IEEE Transactions on Pattern Analysis and Machine Intelligence, vol. 32, no. 4, pp. 722-732, 2008. doi: https: //doi.org/10.1109/tpami.2008.300

[15] X. Zhang, M. Chen, and X. Zhan, "A combined approach to single-camera-based lane detection in driverless navigation," in IEEE/ION Position, Location and Navigation Symposium (PLANS), California, CA, 2018.

[16] J. Deng and Y. Han, "A real-time system of lane detection and tracking based on optimized RANSAC B-spline fitting," in Proceedings of the 2013 Research in Adaptive and Convergent Systems, Quebec, Canada, 2013.

[17] Z. Kim, "Robust lane detection and tracking in challenging scenarios," IEEE Transactions on Intelligent Transportation Systems, vol. 9, no. 1, pp. 16-26, 2008. doi: https://doi.org/10.1109/tits.2007.908582

[18] S. Perreault and P. Hebert, "Median filtering in constant time," IEEE Trans Image Processing, vol. 16, no. 9, pp. 2389-2394, 2007. doi: https://doi.org/10.1109/tip.2007.902329

[19] A. Borkar, M. Hayes, and M. T. Smith, "A novel lane detection system with efficient ground truth generation," IEEE Transactions on Intelligent Transportation Systems, vol. 13, no. 1, pp. 365-374, 2011. doi: https://doi.org/10.1109/tits. 2011.2173196 